Canad. Math. Bull. Vol. 23 (1), 1980

\title{
AUSLANDER-REITEN SEQUENCES FOR "NICE" TORSION THEORIES OF ARTINIAN ALGEBRAS
}

\author{
BY \\ K. W. ROGGENKAMP
}

Let $\mathfrak{f}$ be a field and $\mathfrak{A}$ a finite dimensional $\mathfrak{k}$-algebra. Auslander-Reiten sequences $[A R]$ play a fundamental rôle in the representation theory of $\mathfrak{A}$; in particular, they can be used to construct new indecomposable modules from known ones. For the latter reason I think it worthwile to point out certain torsion theories $\mathfrak{I}$ on the category of $\mathfrak{U}$-modules, such that the category of $\mathfrak{T}$-torsionfree modules has Auslander-Reiten sequences; thus giving another construction of indecomposable modules. It should be noted, that these Auslander-Reiten sequences are different from the ordinary ones.

The impetus to the following observations came from discussions with C. M. Ringel [RR2].

Let $\mathfrak{I}$ be a hereditary torsion theory on $\mathfrak{A}$, generated by the simple $\mathfrak{A}$ modules $S_{1}^{\prime}, \ldots, S_{n}^{\prime}$ (cf. [S]); i.e. the $\mathfrak{I}$-torsionfree modules are the modules $U \in \mathfrak{M}_{\mathfrak{f}}$ - the category of finitely generated left $\mathfrak{A}$-modules-such that the socle of $U-\operatorname{Soc}(U)$ - has no direct summand in $\left\{S_{1}^{\prime}, \ldots, S_{n}^{\prime}\right\}$. If $S_{1}, \ldots, S_{t}$ are the $\mathfrak{I}$-torsionfree simple $\mathfrak{A}$-modules, we denote by $E_{i}=E\left(S_{i}\right), 1=i=t$, the injective envelope of $S_{i}$. The $\mathfrak{I}$-torsionfree $\mathfrak{A}$-modules then can be described as those modules, whose injective envelope decomposes into a direct sum of $E_{i}$ 's. We denote the full subcategory of the torsion free $\mathfrak{A}$-modules by $\mathfrak{X}$. The complete ring of quotients of $\mathfrak{A}$ with respect to $\mathfrak{I}$ is then $\mathfrak{L}=$ $\operatorname{End}_{\operatorname{End}\left(\oplus_{i=1}^{i} E\right)}\left(\bigoplus_{i=1}^{t} E_{i}\right)$, and we have a natural homomorphism

$$
\mathfrak{f}: \mathfrak{A} \rightarrow \mathfrak{Q} .
$$

In order to make our construction work, we have to make the following assumption on:

(1) (i) $\operatorname{End}_{\mathscr{Y}}\left(E_{i}\right)=\mathfrak{f}_{i}$ is a skewfield, $1 \leq i \leq t$,

(ii) $\operatorname{Hom}_{\mathfrak{r}}\left(E_{i}, E_{j}\right)=0$ for $1 \leq i, j \leq t, i \neq j$.

(2) REMARK. The above conditions make sure that

(i) $\mathfrak{L}$ is a semisimple $\mathfrak{t}$-algebra,

(ii) for every $U \in \mathfrak{X}$, the modules of quotients of $U$ with respect to $\mathfrak{I}$ is $E(U)$, the injective envelope of $U$; in particular, $\mathfrak{L}$ is injective as left $\mathfrak{U}$-module (cf. [S, p. 202, 2.3]). 
On the other hand, given any homomorphism of $\mathfrak{k}$-algebras

$$
\kappa: \mathfrak{U} \rightarrow \mathfrak{Q}
$$

such that $\mathfrak{L}$ is semisimple and $\mathfrak{L}$ is an injective $\mathfrak{A}$-module, then this homomorphism is induced by a torsion theory satisfying (1).

(3) ExAmple. Let $\mathfrak{A}$ be the tensoralgebra of the graph $\rightarrow \underbrace{7}_{7}$; i.e.

$$
\mathfrak{A}=\left\{\left(\begin{array}{cccc}
\mathfrak{f}_{1} & \mathfrak{f}_{1} & \mathfrak{f}_{1} & \mathfrak{f}_{1} \\
0 & \mathfrak{f}_{1} & \mathfrak{f}_{1} & \mathfrak{f}_{1} \\
0 & 0 & \mathfrak{f}_{1} & \mathfrak{f}_{1} \\
0 & 0 & 0 & \alpha
\end{array}\right)\left(\begin{array}{cccc}
\mathfrak{f}_{2} & \mathfrak{f}_{2} & \mathfrak{f}_{2} & \mathfrak{f}_{2} \\
0 & \mathfrak{f}_{2} & \mathfrak{f}_{2} & \mathfrak{f}_{2} \\
0 & 0 & \mathfrak{f}_{2} & \mathfrak{f}_{2} \\
0 & 0 & 0 & \alpha
\end{array}\right), \alpha \in \mathfrak{f}\right\}, \mathfrak{f}_{1}=\mathfrak{f}_{2}=\mathfrak{f} .
$$

We choose

$$
S_{1}=\left(\begin{array}{c}
\mathfrak{f}_{1} \\
\mathfrak{f}_{1} \\
0 \\
0
\end{array}\right) /\left(\begin{array}{c}
\mathfrak{f}_{1} \\
0 \\
0 \\
0
\end{array}\right), \quad S_{2},\left(\begin{array}{c}
\mathfrak{f}_{2} \\
\mathfrak{f}_{2} \\
0 \\
0
\end{array}\right) /\left(\begin{array}{c}
\mathfrak{f}_{2} \\
0 \\
0 \\
0
\end{array}\right)
$$

then

$$
E\left(S_{1}\right)=\left(\begin{array}{l}
\mathfrak{f}_{1} \\
\mathfrak{f}_{1} \\
\mathfrak{f}_{1} \\
\mathfrak{f}_{1}
\end{array}\right) /\left(\begin{array}{c}
E_{1} \\
0 \\
0 \\
0
\end{array}\right) \text { and } E\left(S_{2}\right)=\left(\begin{array}{c}
\mathfrak{f}_{2} \\
\mathfrak{f}_{2} \\
\mathfrak{f}_{2} \\
\mathfrak{f}_{2}
\end{array}\right) /\left(\begin{array}{c}
\mathfrak{f}_{2} \\
0 \\
0 \\
0
\end{array}\right),
$$

and $\mathfrak{Q}=\left(\mathfrak{f}_{1}\right)_{3} \Pi\left(\mathfrak{f}_{2}\right)_{3} ;$ moreover, $\kappa: \mathfrak{A} \rightarrow \mathfrak{Q}$ has

$$
\operatorname{Im}(\kappa)=\left\{\left(\begin{array}{ccc}
\mathfrak{f}_{1} & \mathfrak{f}_{1} & \mathfrak{f}_{1} \\
0 & \mathfrak{f}_{1} & \mathfrak{f}_{1} \\
0 & 0 & \alpha
\end{array}\right)\left(\begin{array}{ccc}
\mathfrak{f}_{2} & \mathfrak{f}_{2} & \mathfrak{f}_{2} \\
0 & \mathfrak{f}_{2} & \mathfrak{f}_{2} \\
0 & 0 & \alpha
\end{array}\right), \alpha \in \mathfrak{f}\right\} .
$$

(4) Proposition. $\mathfrak{X}$ has enough projective and injective objects.

Proof. Let $\mathfrak{a}=\operatorname{Ker}(\boldsymbol{\kappa})$, then the projective $\mathfrak{A} / \mathfrak{a}$-modules are precisely the projective objects in $\mathfrak{X}$. In order to construct the injective objects in $\mathfrak{X}$, we have to make a detour.

Let $R=\mathfrak{f} X \rrbracket$ be the ring of formal power series over $\mathfrak{f}$, and let $K$ be the quotient field of $R$. Then

$$
\Gamma=R \otimes_{\mathrm{f}} \mathfrak{R}
$$

is a hereditary $R$-order in $A=K \oplus_{R} \Gamma$; moreover, $\Gamma$ has exactly $t$ nonisomorphic indecomposable lattices, one for each simple $\mathfrak{L}$-module. Let $\Lambda$ be the pullback of the diagram

$$
\begin{array}{ccc}
\mathfrak{A} / \mathfrak{a} & \hookrightarrow & \mathbb{Q} \\
\uparrow & \uparrow \\
\vdots & & \uparrow \\
\Lambda & \Gamma
\end{array}
$$


If $I=\operatorname{Ker}(\Gamma \rightarrow \mathfrak{Q})$, then $I$ is a two-sided $\Gamma$-ideal and $\Lambda / I \cong \mathfrak{A} / \mathfrak{a}$. Since $I \subset \operatorname{rad}(\Lambda)$, we can lift projective modules, and so $\Lambda$ has as many non-isomorphic indecomposable projective modules as has $\mathfrak{A} / \mathfrak{a}$. We put $\overline{\mathfrak{U}}=\mathfrak{Q} / \mathfrak{a}$. Let $I_{1}, \ldots, I_{m}$ be the injective $\Lambda$-lattices; i.e. injective objects in ${ }_{\Lambda} \mathfrak{M}^{0}$, the category of left $\Lambda$-lattices. Then these are of the form $\operatorname{Hom}_{R}(Q, R)$, for $Q$ an indecomposable projective right $\Lambda$-module.

(6) The modules $I_{j} / I I_{j}, i \leq j \leq m$, are the injective objects in $\mathfrak{X}$.

Proof of (6). The embedding $I_{j} \rightarrow \Gamma I_{j}$ induces an embedding

$$
I_{j} / I I_{j} \rightarrow \Gamma I_{j} / I_{j},
$$

however, $\Gamma I_{j} / I_{j}$ is a $\mathfrak{L}$-module; whence injective over $\mathfrak{A}$, and so $I_{j} / I_{j}$ is in $\mathfrak{X}$. Using the injectivity of $I_{j}$ as $\Lambda$-lattice, it is easily seen that $I_{j} / I_{j}$ is an indecomposable injective object in $\mathfrak{X}$, if one observes that reduction modulo $I$ is an exact functor (cf. [R2]), and that reduction modulo $I$ is a representation equivalence between ${ }_{\Lambda} \mathfrak{M}^{0}$ and $\mathfrak{X}$ (cf. [RR1]).

EXAMPLE (3) continued. $\Gamma=\left(R_{1}\right)_{3} \Pi\left(R_{2}\right)_{3}$ and

$$
\Lambda=\left\{\left(\begin{array}{ccc}
R_{1} & R_{1} & R_{1} \\
X R_{1} & R_{1} & R_{1} \\
X R_{1} & X R_{1} & \alpha
\end{array}\right)\left(\begin{array}{ccc}
R_{2} & R_{2} & R_{2} \\
X R_{2} & R_{2} & R_{2} \\
X R_{2} & X R_{2} & \alpha+X R_{2}
\end{array}\right), \alpha \in R\right\} \quad R_{1}=R_{2}=R .
$$

The injective objects in $\mathfrak{X}$ are

$$
\left(\begin{array}{l}
\mathfrak{f}_{1} \\
\mathfrak{f}_{1} \\
\mathfrak{f}_{1}
\end{array}\right),\left(\begin{array}{l}
\mathfrak{f}_{2} \\
\mathfrak{f}_{2} \\
\mathfrak{f}_{2}
\end{array}\right),\left(\begin{array}{l}
\mathfrak{f}_{1} \\
0 \\
0
\end{array}\right),\left(\begin{array}{l}
\mathfrak{f}_{2} \\
0 \\
0
\end{array}\right),\left\{\left(\begin{array}{l}
\mathfrak{f}_{1} \\
\mathfrak{f}_{1} \\
\alpha
\end{array}\right)\left(\begin{array}{l}
\mathfrak{f}_{2} \\
\mathfrak{f}_{2} \\
\alpha
\end{array}\right), \alpha \in \mathfrak{f}\right\} .
$$

It should be noted that not all of these modules are injective $\overline{\mathfrak{A}}$-modules.

(7) Definitions. (i) An exact sequence of objects in $\mathfrak{X}$ is said to be an Auslander-Reiten sequence

$$
\varepsilon: 0 \rightarrow M \stackrel{\varphi}{\rightarrow} E \stackrel{\leftrightarrow}{\rightarrow} N \rightarrow 0, \text { if }
$$

a.) $\varepsilon$ is not split exact,

B.) $M$ and $N$ are indecomposable,

$\gamma$.) given any homomorphism $\alpha: X \rightarrow M(\beta: M \rightarrow Y), X(Y) \in \mathfrak{X}$ such that $\alpha$ is not a split epimorphism ( $\beta$ is not a split monomorphism), then there exists $\sigma: X \rightarrow E(\tau: E \rightarrow Y)$ with $\alpha=\sigma \psi(\beta=\varphi \tau)$.

(ii) $\mathfrak{X}$ is said to have Auslander-Reiten sequences, if given any $N \in \mathfrak{X}, N$ indecomposable not projective in $\mathfrak{X}(M \in \mathfrak{X}, M$ indecomposable not injective in $\mathfrak{X})$, then there exists an Auslander-Reiten sequence

$$
0 \rightarrow M \rightarrow E \rightarrow N \rightarrow 0 \text {. }
$$


By (i) this sequence is uniquely determined up to isomorphism-if it exists.

We write $M=\Delta_{\mathfrak{X}}^{+}(N)$ and $N=\Delta_{\mathfrak{X}}^{-}(M)$, and define $\Delta_{\mathfrak{X}}^{ \pm s}$ inductively.

(9) Proposition. $\mathfrak{X}$ has Auslander-Reiten sequences.

Proof. We shall use the notation of (4). If $N$ is indecomposable nonprojective in $\mathfrak{X}$, then there exists an indecomposable non-projective $\Lambda$-lattice $L$ with $L / I L \cong N$. $\wedge_{\Lambda} \mathfrak{M}^{0}$ has Auslander-Reiten sequences [A, R1, e.a.], so we choose one

$$
0 \rightarrow L^{\prime} \rightarrow L_{0} \rightarrow L \rightarrow 0 .
$$

Since reduction modulo $I$ is exact and preserves non-split sequences, one sees that

$$
0 \rightarrow L^{\prime} / I L^{\prime} \rightarrow L_{0} / I L_{0} \rightarrow N \rightarrow 0
$$

is an Auslander-Reiten sequence in $\mathfrak{X}$. A similar construction is done if $M$ is indecomposable non-injective in $\mathfrak{X}$. (Observe that the injective objects in $\mathfrak{X}$ are in bijection with the injective $\Lambda$-lattices.)

It is possible to give an explicit description of the Auslander-Reiten sequences in $\mathfrak{X}$, similar to the one given in [R3].

We conclude with some remarks on hereditary $\mathfrak{f}$-algebras.

(10) If $\mathfrak{U}$ is a hereditary $\mathfrak{f}$-algebra and $\mathfrak{I}$ a torsiontheory satisfying (1), then $\mathfrak{U} / \mathfrak{a}$ is again a hereditary $\mathfrak{f}$-algebra; in fact, $\mathfrak{a}$ is the torsion submodule of $\mathfrak{A}$, and so $\mathfrak{a}$ is the maximal left $\mathfrak{U}$-ideal which has composition factors only in $\left\{S_{1}^{\prime}, \ldots, S_{n}^{\prime}\right\}$. In order to see that $\mathfrak{U} / \mathfrak{a}$ is hereditary, let $P / \mathfrak{a} P$ be a projective $\mathfrak{U} / \mathfrak{a}$-module, and let $U$ be an $\mathfrak{A} / \mathfrak{a}$-submodule of $P / \mathfrak{a} P$. We form the pullback

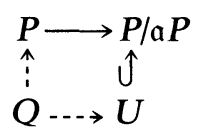

Then $Q$ is a projective $\mathfrak{A}$-module, and it remains to show that a $Q$ is its torsion submodule. But a $Q$ is the kernel of the map

$$
Q \rightarrow \mathfrak{\oplus _ { \mathfrak { Y } }} Q
$$

our torsion theory being perfect, and so $a Q=a P$, and $U$ is a projective $\mathfrak{U} / \mathfrak{a}$-module.

Modifying the proof of [AP] one thus obtains (cf. [R3]);

(11) Let $\mathfrak{A}$ be a hereditary $\mathfrak{f}$-algebra and $\mathfrak{I}$ a torsion theory satisfying (1), then the following are equivalent for the category $\mathfrak{X}$ :

(i) $\mathfrak{X}$ has a finite number of non-isomorphic indecomposable objects.

(ii) For every indecomposable object $U \in \mathfrak{X}$ there exists an $s \in \mathbb{N}$ with $U \cong \Delta_{\mathfrak{X}}^{-s}(P)$, where $P$ is indecomposable projective in $\mathfrak{X}$. 
Finally we turn to the situation where $\mathfrak{A}$ is the tensoralgebra of a $\mathfrak{f}$-species with valued graph $\subseteq$.

(12) We say that an oriented graph $G$ with valuation and without oriented loops is reducible, if there exists an edge $a \stackrel{(1,1)}{\longrightarrow} b$ such that the graph $G \backslash\langle a \stackrel{(1,1)}{\longrightarrow} b\rangle$ which is obtained from $G$ by removing the edge $a \stackrel{(1,1)}{\longrightarrow} b$ is the disjoint union $G^{\prime} \cup A_{s}$ of some graph $G^{\prime}$ and a second graph of type $A_{s}$, in such a way that $a$ is a sink in $G^{\prime}$ and $b$ is a sink in $A_{s}$. We then denote by $G_{a=b}$ the graph obtained from $G$ by identifying $a$ and $b$ and omitting the edge between them, and we say that $G_{a=b}$ is obtained from $G$ by reduction.

(13) Let $\mathfrak{A}$ be the tensoralgebra of a $\mathfrak{f}$-species for $\mathfrak{S}$; then $\mathfrak{A} / \mathfrak{a}$ is the tensoralgebra of a $\mathfrak{f}$-species corresponding to a graph $G$. The following statements are equivalent:

(i) $\mathfrak{X}$ has a finite number of non-isomorphic indecomposable objects,

(ii) $G$ can be reduced (by the process described in (12)) to a Dynkin diagram.

This statement is proved in [RR2] under nearly the same hypotheses.

\title{
REFERENCES
}

[A] Auslander, M., Existence theorems for almost split sequences, Oklahoma Ring theory conference, March 1976.

- , Representation theory of Artin algebras I, II, Comm. Algebra, 1, 177-268 (1974), 1, 239-294 (1975).

[AP] Auslander, M. and I. M. Platzeck, Representation theory of hereditary Artin algebras, Preprint 1976.

[AR] Auslander, M. and I. Reiten, Representation theory of Artin algebras III, Comm. Algebra 3, 239-294 (1975).

[DR] Dlab, V. and C. M. Ringel, Indecomposable representations of graphs and algebras, Memoir Am. Math. Soc. 173, 1976.

[R1] Roggenkamp, K. W., The construction of almost split sequences for integral group rings and orders, Comm. Algebra 5, 1363-1373.

[R2] - Some examples of orders of global dimension two, Math. Z. 154, 225-238 (1977).

- Orders of global dimension two, Math. Z. 160, 63-67, (1978).

[R3] - Indecomposable representations of orders of global dimension two II, to appear $\mathbf{J}$. Algebra.

[RR1] Ringel, C. M. and K. W. Roggenkamp, Diagrammatic methods in representation theory of orders, to appear J. Algebra.

[RR2] - Indecomposable representations of orders of global dimension two I, to be published.

[S] Stenström, B., Rings of quotients, Grundl. Math. 217, Springer-Verlag 1975.

\author{
MATHEMATISCHES INSTITUT B \\ UNIVERSITAT STUTTGART \\ 7 STUTTGart 1 , \\ Postfach 560, West Germany
}

